\title{
THE SHIFTED CLASSICAL CIRCULANT AND SKEW CIRCULANT SPLITTING ITERATIVE METHODS FOR TOEPLITZ MATRICES
}

\author{
ZHONGYUN LIU*, XIAORONG QIN *, NIANCI WU*, AND YULIN ZHANG ${ }^{\dagger}$
}

\begin{abstract}
It is known that every Toeplitz matrix $T$ enjoys a circulant and skew circulant splitting (denoted by CSCS), see [8, 17], i.e., $T=C-S$ with $C$ a circulant matrix and $S$ a skew circulant matrix. Based on the variant of such a splitting (also referred to as CSCS), we first develop classical CSCS iterative methods and then introduce shifted CSCS iterative methods for solving hermitian positive definite Toeplitz systems in this paper. The convergence of each method is analyzed. Numerical experiments show that the classical CSCS iterative methods work slightly better than the Gauss-Seidel (GS) iterative methods if the CSCS is convergent, and that there is always a constant $\alpha$ such that the shifted CSCS iteration converges much faster than the Gauss-Seidel iteration, no matter whether the CSCS itself is convergent or not.
\end{abstract}

Key words. Hermitian positive definite, CSCS Splitting, Gauss-Seidel splitting, Iterative method, Toeplitz matrix.

AMS subject classifications. 15A23, 65F10, 65F15.

1. Introduction. Concerned with the classical iterative solution to a large linear system of equations

$$
T x=b,
$$

where $b \in \mathbb{C}^{n}, T \in \mathbb{C}^{n \times n}$ is an hermitian positive definite (HPD) Toeplitz matrix, which has an extensive applications and has intrigued the researchers for decades [7].

Recall that the representation $A=M-N$ is called a splitting of $A$, if $\mathrm{M}$ is nonsingular. The classical iterative methods for solving $A x=b$ can be described as

$$
M x^{(k+1)}=N x^{(k)}+b \quad k=0,1, \cdots
$$

where $x^{(0)}$ is an initial approximation to the solution of $A x=b$.

It is well-known that the method (1.2) converges for any initial vector $x_{0}$ if and only if the spectral radius $\rho(H)<1$, where $H=M^{-1} N$ is the iteration matrix.

\footnotetext{
* School of Mathematics and Statistics, Changsha University of Science and Technology, Changsha 410076, P. R. China (liuzhongyun@263.net).

†Centro de Matemática, Universidade do Minho, 4710-057 Braga, Portugal (zhang@math. uminho.pt).
} 
The classical iterative methods for solving large linear system of equations (1.1) require efficient splittings of the coefficient matrix $A$. That is to say that $M^{-1}$ is easy to obtain and $\rho(H)<1$. Now, these techniques are rarely used separately. However, when combined with the more efficient methods, they can be quite successful, see for example, $[4,6,8,19]$ and references therein. Moreover, there are a few application areas where variations of these methods are still quite popular, see for instance $[2,3$, $5,10,13,15,17,16]$.

If $A$ is decomposed into the following form

$$
A=D-E-F
$$

where $D,-E$ and $-F$ are the matrices consisting of diagonal entries, strictly lower triangular part, and strictly upper triangular part of $A$, respectively. It is always assumed that $D \neq 0$. Then one can obtain:

- the Jacobi splitting by taking $M=D$;

- Gauss-Seidel splitting by taking $M=D-E$;

- the successive over relaxation(SOR) splitting by taking $M=\frac{1}{\omega}(D-\omega E)$,

- the symmetric successive over relaxation(SSOR) splitting by taking $M=$ $\frac{1}{\omega(2-\omega)}(D-\omega E) D^{-1}(D-\omega F)$.

The matrices $M=D$ and $M=\frac{1}{\omega(2-\omega)}(D-\omega E) D^{-1}(D-\omega F)$ are usually referred as to Jacobi and SSOR preconditioners for preconditioned conjugate gradient method (PCG) and preconditioned generalized minimum residual method (PGMRES).

In this paper we consider $T=\left(t_{i j}\right)_{n \times n}$ in (1.1) being an hermitian Toeplitz matrix defined by $t_{i j}=t_{i-j}$ and $t_{i j}=\bar{t}_{j i}$.

There are two main types of methods for solving Toeplitz systems: direct methods and iterative methods. The complexity of fast direct Toeplitz solvers is $O\left(n \log ^{2} n\right)$, see e.g., $[1,11]$. What's more, the stability of these fast direct algorithms is still in question. Because of these stability problems, considerable attention has recently been given to iterative methods for solving Toeplitz systems, for a early review, see [7].

It is known that a Toeplitz matrix $T$ always possesses a circulant and skewcirculant splitting (CSCS)

$$
T=C_{\beta}-S_{\gamma},
$$


where

$$
C_{\beta}=\left(\begin{array}{cccc}
\beta & \frac{t_{-1}+t_{n-1}}{2} & \ldots & \frac{t_{1-n}+t_{1}}{2} \\
\frac{t_{1}+t_{1-n}}{2} & \beta & \ldots & \frac{t_{-n}+t_{2}}{2} \\
\vdots & \vdots & \ddots & \vdots \\
\frac{t_{n-1}+t_{-1}}{2} & \frac{t_{n-2}+t_{-2}}{2} & \ldots & \beta
\end{array}\right)
$$

is a circulant matrix, and

$$
S_{\gamma}=-\left(\begin{array}{cccc}
\gamma & \frac{t_{-1}-t_{n-1}}{2} & \cdots & \frac{t_{1-n}-t_{1}}{2} \\
\frac{t_{1}-t_{1-n}}{2} & \gamma & \cdots & \frac{t_{-n}-t_{2}}{2} \\
\vdots & \vdots & \ddots & \vdots \\
\frac{t_{n-1}-t_{-1}}{2} & \frac{t_{n-2}-t_{-2}}{2} & \cdots & \gamma
\end{array}\right)
$$

is a skew-circulant matrix, the parameters $\beta, \gamma$ satisfy $\beta+\gamma=t_{0}$.

If taking $\beta=\gamma=\frac{t_{0}}{2}$, then the splitting (1.4) becomes one used by $\mathrm{Ng}$ in [17], which resulted in a so-called CSCS iterative method consisting of two half-step iterations, for nonhermitian positive definite Toeplitz systems, analogously to the classical alternating direction implicit (ADI) iteration for solving partial differential equations. Moreover, based on this splitting, Chan and Jin in [8] developed some circulant and skew-circulant preconditioners for skew hermitian type Toeplitz systems.

In this paper, we propose an classical iterative solvers for hermitian positive define Toeplitz systems based on the circulant /skew-circulant splitting iteration by always taking $\beta>\gamma$, and it is as follows.

The Classical CSCS iteration: Given an initial guess $x^{(0)}$, for $k=0,1,2, \cdots$ until $\left\{x^{(k)}\right\}$ converges, compute

$$
C_{\beta} x^{(k+1)}=S_{\gamma} x^{(k)}+b,
$$

where $C_{\beta}$ is assumed to be nonsingular.

We remark here that the main operations in (1.5) are matrix-vector products $C_{\beta}^{-1} \mathbf{u}$ and $S_{\gamma} \mathbf{v}$. Since circulant matrices can be diagonalized by the discrete Fourier matrix $F$ and the skew-circulant matrices can be diagonalized by the diagonal times discrete Fourier matrix $\hat{F}$, i.e.,

$$
C_{\beta}=F^{*} \Lambda F \quad \text { and } \quad S_{\gamma}=\hat{F}^{*} \Sigma \hat{F}
$$

where $\Lambda$ and $\Sigma$ are diagonal matrices holding the eigenvalues of $C_{\beta}$ and $S_{\gamma}$ respectively, the exact solutions with circulant matrices can be obtained by using 8 fast Fourier transforms (FFTs) of $n$-vectors. We emphasize that the use of circulant and 
skew-circulant matrices for solving Toepltiz systems allows the use of FFT throughout the computations, and FFT is highly parallelizable and has been implemented on multiprocessors efficiently. The proposed method is well-adapted for parallel computing.

Of course, we can use the Gauss-Seidel iterative method for solving hermitian positive define Toeplitz systems. If $T$ is split as $(D-L)-L^{*}$, then the Gauss-Seidel iteration is as follows.

The Classical GS iteration: Given an initial guess $x^{(0)}$, for $k=0,1,2, \cdots$ until $\left\{x^{(k)}\right\}$ converges, compute

$$
(D-L) x^{(k+1)}=L^{*} x^{(k)}+b,
$$

where $D-L$ is the lower triangular Toeplitz matrix and $L^{*}$ is the strictly upper triangular Toeplitz matrix.

The main operations in (1.7) is to calculate $(D-L)^{-1},(D-L)^{-1} \mathbf{u}$ and $L^{*} \mathbf{v}$. A fast algorithm in $[9,14]$ for computing $(D-L)^{-1}$ requires about 10 FFTs of $n$-vectors. Furthermore, to compute $(D-L)^{-1} \mathbf{u}$ and $L^{*} \mathbf{v}$ needs 6 FFTs of $2 n$-vectors, see, e.g., [7]. That is to say that the exact solutions with lower triangular Toeplitz matrices can be obtained by using 22 FFTs of $n$-vectors. This means that our classical CSCS iteration ensures significant savings, as compared to the classical GS iteration, at each iterative step.

The organization of this paper is as follows. In next section, we first recall some preliminaries, then study the convergence of the classical CSCS iteration and finally propose a new CSCS iteration with a shift. Numerical experiments are presented in section 3 to show the effectiveness of our methods. A brief conclusion is also drawn in section 4 and the acknowledgements are followed in last section.

2. The shifted CSCS iteration. In this section, we first review some known results needed in the remaining parts of this paper, then introduce the shifted CSCS iterative methods for Toeplitz system (1.1), finally convergence results are given for each scheme.

2.1. Preliminaries. A matrix $A$ is said to be positive definite if $x^{*} A x>0$ for $\forall x \in \mathbb{C}^{n}, x \neq 0$.

Regarding splittings of the positive definite matrix $A$, we need the following definition and theorems which can be found in $[12,15,16,18]$.

Definition 2.1. The splitting $A=M-N$ is called P-regular if $\tilde{T}=M^{*}+N$ is positive definite. 
TheOREm 2.2. Let $A=M-N$ be an hermitian positive definite matrix, where $M$ is a invertible hermitian matrix. Then $\rho\left(M^{-1} N\right)<1$ if and only if the splitting $A=M-N$ is P-regular.

Theorem 2.3. Let $A$ be hermitian, and let the splitting $A=M-N$ be P-regular. Then $\rho\left(M^{-1} N\right)<1$ if and only if $A$ is positive definite.

Theorem 2.4. (Weyl's theorem). Let $A, B \in \mathbb{C}^{n \times n}$ be Hermitian and the eigenvalues $\lambda_{i}(A), \lambda_{i}(B), \lambda_{i}(A+B)$ of $A, B$ and $A+B$ be arranged in an increasing order. Then, for each $i=1,2, \ldots, n$, we have

$$
\text { (i) } \quad \lambda_{i}(A)+\lambda_{1}(B) \leq \lambda_{i}(A+B) \leq \lambda_{i}(A)+\lambda_{n}(B)
$$$$
\text { (ii) } \quad \lambda_{1}(A)+\lambda_{i}(B) \leq \lambda_{i}(A+B) \leq \lambda_{n}(A)+\lambda_{i}(B)
$$

2.2. The classical CSCS iteration with a shift. Notice first that if $T$ is hermitian, then the matrices $C_{\beta}$ and $S_{\gamma}$ defined as in (1.4) are also hermitian. In this case their eigenvalues $\lambda_{i}\left(C_{\beta}\right)$ and $\lambda_{i}\left(S_{\gamma}\right)$ are all real, can be arranged in an increasing order and obtained easily by using 2 FFTs. As a natural consequence of Theorem 2.2 , we have the following results.

Lemma 2.5. Let $T$ be an hermitian positive definite Toeplitz matrix, $T=C_{\beta}-S_{\gamma}$ be the circulant and skew-circulant splitting. If the splitting $T=C_{\beta}-S_{\gamma}$ is P-regular, then $\rho\left(C_{\beta}^{-1} S_{\gamma}\right)<1$.

Now, we can establish a sufficient condition on the convergence of the classical CSCS iteration.

THEOREM 2.6. Let $T$ be an hermitian positive definite Toeplitz matrix, $T=$ $C_{\beta}-S_{\gamma}$ be the circulant and skew-circulant splitting, $\lambda_{1}\left(C_{\beta}\right)$ and $\lambda_{1}\left(S_{\gamma}\right)$ be the smallest eigenvalues of $C_{\beta}$ and $S_{\gamma}$, respectively. If $\lambda_{1}\left(C_{\beta}\right)+\lambda_{1}\left(S_{\gamma}\right)>0$, then $\rho\left(C_{\beta}^{-1} S_{\gamma}\right)<1$ i.e., the iteration (1.5) converges to the exact solution $x^{\star}$ of the linear system of equations (1.1).

Proof. From the hypothesis and by Theorem 2.4, we have that

$$
\lambda_{i}\left(C_{\beta}^{*}+S_{\gamma}\right) \geq \lambda_{1}\left(C_{\beta}\right)+\lambda_{1}\left(S_{\gamma}\right)>0 .
$$

This means that $\tilde{T}=C_{\beta}^{*}+S_{\gamma}$ is hermitian positive definite. Thus the splitting $T=C_{\beta}-S_{\gamma}$ is P-regular. By Lemma 2.5 , we have $\rho\left(C_{\beta}^{-1} S_{\gamma}\right)<1$ immediately.

We observe here that the splitting $T=C_{\beta}-S_{\gamma}$ is not always P-regular for given $\beta$ and $\gamma$, even if $\beta>\gamma$ (the assumption $\lambda_{1}\left(C_{\beta}\right)+\lambda_{1}\left(S_{\gamma}\right)>0$ implies $\beta>\gamma$ ). In this case, we introduce a positive parameter $\alpha$ and consider the following splitting

$$
T=\hat{C}_{\beta}-\hat{S}_{\gamma},
$$


where $\hat{C_{\beta}}=\alpha I+C_{\beta}$ and $\hat{S_{\gamma}}=\alpha I+S_{\gamma}$, which are obtained from $C_{\beta}$ and $S_{\gamma}$ by shifting $\alpha I$, respectively. We refer to the splitting (2.1) as the shifted CSCS and its corresponding iteration as the shifted CSCS iteration. It is as follows.

The shifted CSCS iteration: Given an initial guess $x^{(0)}$, for $k=0,1,2, \cdots$, until $\left\{x^{(k)}\right\}$ converges, compute

$$
\hat{C}_{\beta} x^{(k+1)}=\hat{S}_{\gamma} x^{(k)}+b,
$$

where $\hat{C}_{\beta}$ is nonsingular.

Since $\hat{C}_{\beta}$ and $\hat{S}_{\gamma}$ in (2.1) are circulant and skew circulant matrices respectively, we can fast perform the shifted CSCS iteration by employing DFT, similar to the classical CSCS iteration used.

TheOREM 2.7. Let $T$ be an hermitian positive definite Toeplitz matrix, $T=$ $\hat{C}_{\beta}-\hat{S}_{\gamma}$ be the shifted CSCS defined in (2.1). If the splitting $T=C_{\beta}-S_{\gamma}$ is not P-regular, then there exists a positive constant $\alpha$ such that $\rho\left(\hat{C}_{\beta}^{-1} \hat{S}_{\gamma}\right)<1$ i.e., the iteration (2.2) converges to the exact solution $x^{\star}$ of the linear system of equations (1.1).

Proof. By Theorem 2.2, it suffices to show that there is a constant such that $\tilde{\hat{T}}=\hat{C}_{\beta}{ }^{*}+\hat{S}_{\gamma}$ is positive definite. Note that $\tilde{\hat{T}}=2 \alpha I+\left(C_{\beta}^{*}+S_{\gamma}\right)=2 \alpha I+\tilde{T}$ and $\lambda_{i}(\tilde{\hat{T}})=2 \alpha+\lambda_{i}(\tilde{T}) \geq 2 \alpha+\lambda_{1}(\tilde{T}) \geq 2 \alpha+\lambda_{1}\left(C_{\beta}\right)+\lambda_{1}\left(S_{\gamma}\right)$. Thus, we can take a $\alpha$ such that $\alpha>-\lambda_{1}(\tilde{T}) / 2$, which means that $\tilde{\hat{T}}$ is positive definite. However, $\lambda_{1}(\tilde{T})$ is unknown. Note that $\lambda_{1}\left(C_{\beta}\right)$ and $\lambda_{1}\left(S_{\gamma}\right)$ can be easily obtained. Instead, we can take a $\alpha$ such that $\alpha>-\left[\lambda_{1}\left(C_{\beta}\right)+\lambda_{1}\left(S_{\gamma}\right)\right] / 2$, which also means that $\tilde{\hat{T}}$ is positive definite. i.e, $T=\hat{C}_{\beta}-\hat{S}_{\gamma}$ is P-regular. By Theorem 2.2 , we have $\rho\left(\hat{C}_{\beta}^{-1} \hat{S}_{\gamma}\right)<1$. The proof is thus complete.

Theorem 2.7 tell us that even if the splitting $T=C_{\beta}-S_{\gamma}$ is not P-regular, there is always a constant $\alpha>0$ such that the shifted CSCS iteration converges. At the same time, we observe that the shifted CSCS iteration reduces to the classical CSCS iteration when $\alpha=0$, which is similar to that the SOR iteration reduces to GS iteration when $\omega=1$. That is to say that the parameter $\alpha$ in the shifted CSCS iteration plays the same role as $\omega$ in SOR iteration. Therefore, there does exist an optimal parameter $\alpha^{*}$ such that $\rho\left(\left(\alpha^{*} I+C_{\beta}\right)^{-1}\left(\alpha^{*} I+S_{\gamma}\right)\right) \leq \rho\left(C_{\beta}^{-1} S_{\gamma}\right)$. Hence we can expect that the shifted CSCS iteration has a better convergence rate than the classical CSCS iteration when the splitting $T=C_{\beta}-S_{\gamma}$ is P-regular. The numerical experiments in next section verify the our guess.

3. Numerical examples. All the numerical tests were done on a Founder desktop PC with Pentium dual-core E6700 CPU 3.20 GHz with Matlab 7.4.0.287 (R2007a). 
To verify the effectiveness of our method, several kinds of generating functions were tested and they are as follows

EXAMPLE 3.1. $f=1+x^{2}, b=(1,1, \cdots, 1)^{T}$ and $x^{(0)}=(1,1, \cdots, 1)^{T}$.

EXAmple 3.2. $t_{k}=(1+|k|)^{-p}, b=(1,1, \cdots, 1)^{T}$ and $x^{(0)}=(1,0, \cdots, 0)^{T}$.

EXAmple 3.3. $f=0.1+|x|, b=(1,1, \cdots, 1)^{T}$ and $x^{(0)}=(1,0, \cdots, 0)^{T}$.

EXAmple 3.4. $f=1.1+\cos x, b=(1,1, \cdots, 1)^{T}$ and $x^{(0)}=(1,0, \cdots, 0)^{T}$.

By Theorem 2.3, we know that the Gauss-Seidel iteration of an hermitian positive definite matrix is always convergent. For comparison, we therefore test GS iteration. In all tests, the scalars $\beta=t_{0}$ and $\gamma=0$ in the CSCS (1.4), and the stopping criteria is $\epsilon=\frac{\left\|r^{(k)}\right\|_{2}}{\left\|r^{(0)}\right\|_{2}} \leq 10^{-6}$, where $r^{(k)}$ is the residual vector at the $k$ th iteration. In all tables, $N, n, \mathrm{GS}, \mathrm{CS}$ and $\mathrm{CS}(\alpha)$ mean the number of iteration, the order of the matrix $T$, the Gauss-Seidel iteration, the classical CSCS iteration and the shifted CSCS iteration with the shift $\alpha$, respectively.

The CSCS of $T$ in Example 3.1 is P-regular. So the classical CSCS iteration is convergent. The number of iterations required for convergence is illustrated in Table 3.1 in which we can see our method converges slightly faster than the GS iterative method.

Table 3.1 CS vs GS for Example 3.1

\begin{tabular}{|c|c|c|c|c|c|}
\hline$N$ & 64 & 128 & 256 & 512 & 1024 \\
\hline GS & 23 & 23 & 23 & 23 & 23 \\
\hline CS & 21 & 21 & 21 & 21 & 21 \\
\hline
\end{tabular}

For Example 3.2, we compute the smallest eigenvalues of the matrices $\hat{T}=C^{*}+S$ which are listed in Table 3.2. It indicates that all the splittings of $T$ in the cases of $p=0.8, p=1.0, p=1.2$ and $p=1.4$ are not P-regular.

Table 3.2 The smallest eigenvalues of $\hat{T}$ for Example 3.2

\begin{tabular}{|c|c|c|c|c|}
\hline $\mathrm{p}$ & 128 & 256 & 512 & 1024 \\
\hline 0.8 & -1.2074 & -1.6996 & -2.2389 & -2.8321 \\
\hline 1.0 & -0.4726 & -0.6476 & -0.8020 & -0.9371 \\
\hline 1.2 & -0.0117 & -0.0659 & -0.1023 & -0.1256 \\
\hline
\end{tabular}

We select a $\alpha$ being a small perturbation of $-\left[\lambda_{1}\left(C_{\beta}\right)+\lambda_{1}\left(S_{\gamma}\right)\right] / 2$ (i.e., $0<-\lambda_{1}$ $\left.(\tilde{T}) / 2<\alpha \leq-\left[\lambda_{1}\left(C_{\beta}\right)+\lambda_{1}\left(S_{\gamma}\right)\right] / 2\right)$ such that the shifted CSCS is P-regular. The 
numbers of iterations required for convergence are illustrated in Tables 3.3-3.6. It is clear that the shifted CSCS iterative methods converge much faster than the GS iterative methods for larger $n$.

Table $3.3 \mathrm{CS}(\alpha)$ vs GS for Example 3.2 with $p=0.8$

\begin{tabular}{|c|c|c|c|c|c|}
\hline $\mathrm{N}$ & 64 & 128 & 256 & 512 & 1024 \\
\hline$G S$ & 34 & 43 & 54 & 66 & 82 \\
\hline$C S(\alpha)$ & $34(0.795)$ & $40(1.03)$ & $46(1.32)$ & $52(1.658)$ & $58(2.05)$ \\
\hline
\end{tabular}

Table $3.4 \mathrm{CS}(\alpha)$ vs GS for Example 3.2 with $p=1.0$

\begin{tabular}{|c|c|c|c|c|c|}
\hline $\mathrm{N}$ & 64 & 128 & 256 & 512 & 1024 \\
\hline$G S$ & 26 & 32 & 39 & 45 & 53 \\
\hline$C S(\alpha)$ & $24(0.61)$ & $26(0.730)$ & $28(0.855)$ & $30(0.95)$ & $31(1.03)$ \\
\hline
\end{tabular}

Table $3.5 \operatorname{CS}(\alpha)$ vs GS for Example 3.2 with $p=1.2$

\begin{tabular}{|c|c|c|c|c|c|}
\hline $\mathrm{N}$ & 64 & 128 & 256 & 512 & 1024 \\
\hline$G S$ & 22 & 25 & 29 & 33 & 37 \\
\hline$C S(\alpha)$ & $18(0.46)$ & $19(0.48)$ & $19(0.55)$ & $19(0.63)$ & $19(0.65)$ \\
\hline
\end{tabular}

Table 3.6 $\operatorname{CS}(\alpha)$ vs GS for Example 3.2 with $p=1.4$

\begin{tabular}{|c|c|c|c|c|c|}
\hline $\mathrm{N}$ & 64 & 128 & 256 & 512 & 1024 \\
\hline$G S$ & 18 & 21 & 23 & 25 & 27 \\
\hline$C S(\alpha)$ & $14(0.34)$ & $14(0.35)$ & $14(0.4)$ & $15(0.43)$ & $15(0.445)$ \\
\hline
\end{tabular}

All CSCSs of $T$ in Example 3.3-3.4 are P-regular. So the classical CSCS iterations are naturally convergent. However, after introducing a parameter $\alpha$ (Here, the $\alpha$ is taken to be a small perturbation of $\left.-\left[\lambda_{1}\left(C_{\beta}\right)+\lambda_{1}\left(S_{\gamma}\right)\right] / 2\right)$ ((i.e., $-\lambda_{1}(\tilde{T}) / 2<\alpha$ $\left.\leq-\left[\lambda_{1}\left(C_{\beta}\right)+\lambda_{1}\left(S_{\gamma}\right)\right] / 2\right)$ such that the shifted CSCSs are also P-regular, we find the shifted CSCS iterations work better than the classical CSCS iterations. The numbers of iterations required for convergence are illustrated in Tables 3.7-3.8, respectively.

4. Conclusion. In this paper, we consider the classical iterative solver of the hermitian positive definite Toeplitz linear system of equations $T x=b$. A shifted CSCS iterative method is proposed and its convergence is also discussed. We have shown that there always exists a constant $\alpha$ such that the shifted CSCS is P-regular, even if the classical CSCS is not P-regular. Moreover, if the classical CSCS is P-regular, we may choose a $\alpha$ such that the sifted CSCS iteration has a better convergence rate 
Table 3.7 CS $(\alpha)$ vs GS for Example 3.3

\begin{tabular}{|c|c|c|c|c|c|}
\hline $\mathrm{N}$ & 64 & 128 & 256 & 512 & 1024 \\
\hline $\mathrm{GS}$ & 83 & 96 & 105 & 109 & 112 \\
\hline $\mathrm{CS}(0)$ & 83 & 96 & 104 & 109 & 112 \\
\hline $\mathrm{CS}(\alpha)$ & $43(-0.391)$ & $48(-0.418)$ & $52(-0.42)$ & $53(-0.43)$ & $54(-0.435)$ \\
\hline
\end{tabular}

Table $3.8 \mathrm{CS}(\alpha)$ vs GS for Example 3.4

\begin{tabular}{|c|c|c|c|c|c|}
\hline $\mathrm{N}$ & 64 & 128 & 256 & 512 & 1024 \\
\hline $\mathrm{GS}$ & 40 & 38 & 36 & 34 & 32 \\
\hline $\mathrm{CS}(0)$ & 37 & 35 & 33 & 33 & 30 \\
\hline $\operatorname{CS}(\alpha)$ & $26(-0.155)$ & $25(-0.165)$ & $24(-0.166)$ & $24(-0.17)$ & $24(-0.176)$ \\
\hline
\end{tabular}

than the classical CSCS iteration. Numerical experiments show that the sifted and unshifted CSCS iterations have better convergence behaviors than the classical GS iterations.

We remark here that the sifted classical CSCS iterative method considered in this paper is only for hermitian positive definite Toeplitz linear systems, but this method can be generalized to general hermitian positive definite linear systems.

It is an important and hard task to find the optimal $\alpha$ which strongly depend on the concrete structures and properties of the coefficient matrix $T$ and needs further in-depth study from the viewpoint of both theory and computations.

5. Acknowledgement. The authors would like to thank the supports of the National Natural Science Foundation of China under Grant No. 11371075, the research innovation program of Hunan province for postgraduate students under Grant No. CX2015B374, the Portuguese Funds through FCT-Fundacão para a Ciência, within the Project UID/MAT/00013/2013.

\section{REFERENCES}

[1] G. Ammar and W. Gragg, Superfast solution of real positive definite Toeplitz systems, SIAM J. Matrix Anal. Appl. 9 (1988), pp. 61-76.

[2] Z. Z. Bai, G. H. Golub, and M. K. Ng, Hermitian and skew-hermitian spiltting methods for non-hermitian positive definite linear systems, SIAM J. Matrix Anal. Appl. Vol. 24 (2003), No. 3, pp. 603-626.

[3] Z. Z. Bai, G. H. Golub, L. Z. Lu, and J. F. Yin, Block triangular and skew-hermitian spiltting methods for nonhermitian positive definite systems, SIAM J. Matrix Anal. Appl. Vol. 26 (2005), No.3, pp. 844-863.

[4] Z. Z. Bai, G. H. Golub, and J. Y. Pan, Preconditioned hermitian and skew-hermitian splitting 
methods for non-hermitian positive semidefinite linear systems, Numer. Math., 98 (2004), pp. 1-32.

[5] M. Benzi, A generalization of the hermitian and skew-hermitian spiltting iteration, SIAM J. Matrix Anal. Appl. Vol.31 (2009), No.2, pp. 360-374.

[6] M. Benzi and D. Bertaccini, Block preconditioning of real-valued iterative algorithms for complex linear systems, IMA J. Numer. Anal., 28 (2008), pp. 598-618.

[7] R. Chan and M. Ng, Conjugate gradient methods for Toeplitz systems, SIAM Rev. 38 (1996), pp. 427-482.

[8] R. H. Chan and X-Q J, Circulant and skew-circulant preconditioners for skew-hermitian type Toeplitz systems, BIT 31 (1991), pp. 632-646.

[9] D. Commges and M. Monsion, Fast inversion of triangular Toeplitz matrices, lEEE Transactions on automatic control, 29 (1984), pp. 250-251.

[10] A. Frommer, D.B. Szyld, H-splitting and two-stage iterative methods, Numer. Math. 63 (1992), pp. 345-356.

[11] G. Golub and C. Van Loan, Matrix Computations, Johns Hopkins University Press, Baltimore and London, 3rd edition, 1996.

[12] R. A. Horn and C. R. Johnson, Matrix Analysis, Cambridge University Press, Cambridge, UK, 1985.

[13] P. J. Lanzkron, D. J. Rose and D. B. Syzld, Convergence of nested classical iterative methods for linear systems, Numer. Math. 58 (1991), pp. 685-702.

[14] F. R. Lin, W. K. Ching and M. K. Ng, Fast inversion of triangular Toeplitz matrices, Theoretical Computer Science 315 (2004), pp. 511-523.

[15] Z. Y. Liu, H. B. Wu and L. Lin, The two-stage iterative methods for symmetric positive definite matrices, Applied Math. Comput., 114 (2000), pp. 1-12.

[16] R. Nabben, A note on comparison theorems for splittings and multisplittings of hermitian positive definite matrices, Linear Algebra Appl., 233 (1996), 67-80.

[17] M. K. Ng, Circulant and skew-circulant splitting methods for Toeplitz systems, J. Comput. Applied Math. 159 (2003), pp. 101-108.

[18] J. M. Ortega , Numerical Analysis: A Second Course, Academic Press, New York, 1972.

[19] Y. Saad, Iterative methods for sparse Linear systems, 2nd edition, SIAM, Philadelphia, PA, USA, 2000.

[20] F. B. Weissler, Some remarks concerning iterative methods for linear systems, SIAM. J. Matrix Anal. Appl. 16 (2) (1995) 448-461. 